\title{
Instrumentos para la revitalización y el desarrollo sustentable de poblados de rango menor y de fragmentos urbanos degradados
}

\author{
F. Pini, R. Ferraro, F. Robles, S. Lanari y J. Ticona \\ Con la participación de los alumnos V. Ríos, C. Veliz y D. Otero \\ Instituto de investigaciones del hábitat y el medio ambiente. \\ Facultad de Arquitectura, Diseño, Arte y Urbanismo. Universidad de Morón. \\ E-mail: fpini@unimoron.edu.ar; fopini@yahoo.com.ar
}

\section{Resumen}

Existen en nuestro País, poblados de rango menor (hasta 2000 habitantes) y fragmentos urbanos desintegrados que presentandificultadespara sosteneractividades productivas, equipamientos, servicios y población, consiguientemente, dificultades para crear condiciones adecuadas de vida y con tendencia hacia la desaparición y/o degradación de los mismos, muchos con evidencias de un pasado de esplendor que fue deteriorándose con el tiempo y que, en algunos casos, mantienen el potencial que les permitiría iniciar un proceso de desarrollo sustentable que la revierta, avanzando hacia su revitalización y satisfacción de requerimientos de habitabilidad. Tales situaciones hacen necesario identificar las redes causales e interpretar los procesos que deterioran las condiciones socio-ambientales de habitabilidad y de funcionalidad del hábitat urbano en poblados de rango menor y en fragmentos urbanos degradados, así como identificar modelos de gestión, instrumentos y modalidades de actuación que posibiliten la revitalización y desarrollo sustentable de los mismos -qué hacer, cómo hacer. Para el logro de tales objetivos, se llevó a cabo una investigación aplicada con enfoque cualitativo interpretativo basado en el estudio comparativo de casos, contrastados con el marco teórico, mediante técnica documental y de campo. La muestra presenta coincidencias con antecedentes del marco teórico, pero también diferencias que permiten someter a reconsideración algunas de sus afirmaciones. Los requerimientos de Desarrollo Sustentable: complejidad, sinapsis, integración, cooperación, homeostasis, solidaridad y sinergia, presentan escasa masa crítica, impidiendo dinámicas de transformación deseables y haciendo necesario internalizarlas en la cultura local y en las conductas de los responsables de la gestión territorial. Tal situación requiere identificar fortalezas locales, ventajas, singularidades, potencialidades para la diversificación de actividades, capacidades de diferenciación, y a su vez, con mirada territorial eco-sistémica, lograr que cada poblado se potencie como nodo de una red de comunidades sostenibles organizada según el mix virtuoso de la cultura del desarrollo, cooperación/competencia. Es decir, compensar simplicidad, falta de masa crítica y tiempo para la transformación, mediante la integración complementaria, cooperativacolaborativa, dinámica, de cada uno de ellos, en una red interactiva local, regional y nacional, co-gestionada entre los actores locales y las diferentes escalas de gobierno, y conectada con fuentes de información y conocimiento que los potencien.

Palabras clave: desarrollo local sustentable, políticas territoriales, dignidad social, poblados de rango menor, fragmentos urbanos degradados 


\section{Antecedentes y Situación Actual del tema}

En una nota que el Diario Clarín ${ }^{1}$ realizó el 8/03/2012, a la creadora de la ONG Responde, la geógrafa Margarita Benítez $^{2}$, quedan reflejados parámetros de la situación: "Nacieron hace unos 100 años y muchos están viviendo sus últimos días. Son los pueblos argentinos de menos de dos mil habitantes". "Algunos comenzaron su declive cuando terminó la minería, la esquila de ovejas 0 cualquiera que fuese la actividad económica que había sido su razón de ser. Otros sufrieron la razón más clásica del despoblamiento: el fin del ferrocarril. Todos tienen en común la emigración de sus pobladores hacia ciudades donde no siempre mejoran sus condiciones de vida".

"Las reformas de mercado y los procesos de internacionalización económica y globalización cultural han invalidado las estrategias tradicionales de desarrollo rural" (Micieli3).

"El tema de la infraestructura y las comunicaciones es básico para que un poblado rural pueda funcionar de forma similar a una ciudad de mayor tamaño". (Benítez, op.cit)

"Por otro lado, desde el punto de vista de la educación y capacitación, la cuestión es que han cambiado los modos de producción y en los pueblos sigue existiendo solamente la escuelita primaria. Con este panorama tan deficitario la gente no está en condiciones de plantearse proyectos económicos que terminen siendo un emprendimiento productivo". (Benítez, op. cit.)

Las dificultades para encontrar trabajo que gran parte de esta población rural enfrenta cuando deja el campo por la ciudad, induce a que, en general, se establezcan en sectores urbanos desintegrados, fragmentados y degradados.

${ }^{1} \mathrm{http} / / /$ www.clarin.com/rural/expoagro/pueblos-gentedesaparecieron_0_659934067.html

${ }^{2}$ Licenciada en Geografía y doctora en Sociología. Su tesis doctoral, La Argentina que desaparece, que desarrolló como becaria e investigadora del CONICET entre los años 1991 y 2003, le permitió aproximarse y estudiar la problemática del despoblamiento rural en la Argentina. En 1999 fundó la ONG Responde, dedicada a evitar la extinción de numerosos pueblos de nuestro país. En la actualidad, su labor es reconocida a nivel mundial.

${ }^{3}$ Pueblos Rurales: Localidades en riesgo de desaparición. Blog de la asignatura electiva FADU-UBA Arquitectura Sustentable, cátedra Arq. Adriana Miceli. https://arqsust.wordpress.com/trabajos-practicos/ pueblos-rurales/

\section{Preguntas de Investigación}

¿Qué redes causales y procesos han interactuado como factores deteriorantes de las condiciones de vida de poblados de rango menor?

¿Qué similitudes $y / 0$ interacciones existen entre las condiciones de vida de tales poblados de rango menor y las de fragmentos urbanos degradados?

¿Cuál es el potencial que poseen estos poblados para desencadenar procesos de DLS?

¿Qué recomendaciones y pautas referenciales conceptuales y metodológicas pueden definirse?

\section{Objetivos}

- Identificar las redes causales e interpretar los procesos que deterioran las condiciones del hábitat urbano en poblados de rango menor de Argentina y en fragmentos urbanos degradados del AMBA.

- Identificar modelos de gestión y modalidades de actuación que posibiliten la revitalización y desarrollo sustentable de tales poblados y fragmentos urbanos -qué hacer, cómo hacer-.

- Elaborar recomendaciones a llevar a cabo por la comunidad, las ONGs y la gestión pública.

\section{Hipótesis de la Investigación}

El deterioro de las condiciones de habitabilidad y funcionalidad en poblados de rango menor $y$ en fragmentos urbanos degradados, poseen redes causales y condicionantes con rasgos comunes

Los poblados de rango menor y los fragmentos urbanos degradados, reúnen $y / 0$ conservan condiciones socioambientales y ecosistémicas que propician procesos de desarrollo sustentable

\section{Metodología de Trabajo}

El estudio es una investigación aplicada que presenta un enfoque cualitativo interpretativo y que utiliza el estudio de casos mediante técnica documental y de campo, cuya fuente de información principal estuvo constituida por trabajos de cátedra de Arquitectura 4, Urbanismo 3 y Proyecto Final Integrador, en el marco del Programa por la Dignidad Social de los Pueblos, promovidos por la UM-FADAU desde 2014, así como por publicaciones y documentos en soporte papel 0 digitales. 


\section{Variables de Análisis}

Se sistematizó la información de cada caso de estudio, según las siguientes variables de análisis: 1.-Factores condicionantes locacionales. 2.-Factores condicionantes naturales. 3.-Factores condicionantes históricos. 4.-Factores condicionantes funcionales. 5.-Factores condicionantes socio-culturales. 6.-Factores condicionantes del modelo productivo. 7.-Factores condicionantes externos. 8.-Diagnóstico de situación y de posicionamiento Recomendaciones

\section{Selección de los Casos de Estudio}

Se clasificaron según cuatro modelos territoriales de características homogéneas y se seleccionaron los casos más relevantes. La selección se realizó por muestreo no aleatorio (de juicio 0 empírico) según muestreo subjetivo por decisión razonada.

- Región Noroeste: Mutquin, Catamarca. San Miguel de los Colorados, Jujuy. Yacuy, Salta.

- Región Litoral-Mesopotámica: Carbó, Entre Ríos. Pueblo Liebig, Entre Ríos.

- Provincia de Buenos Aires: Las Marianas. Villars.

- Fragmentos urbanos-CABA: Eje Riachuelo-Comunas 4 y 8. Villa de emergencia 21-24, Barracas.

\section{Estudios Comparativos - Resultados - Conclusiones}

En cada una de las fichas de los poblados y fragmentos urbanos analizados, se enunciaron un diagnóstico y un conjunto de recomendaciones en relación a sus situaciones locales. El estudio muestra coincidencias respecto al marco teórico, y también diferencias que permiten someter a reconsideración algunas de sus afirmaciones. En relación a las condiciones previas necesarias para desencadenar procesos de desarrollo local sustentable -Complejidad, Sinapsis, Sinergia y con ellos, integración, cooperación, solidaridad- la evaluación de los casos concluye que respecto a:

Complejidad: Este requerimiento para el desarrollo local, no verifica en los casos del estudio. En general los poblados se caracterizan por su simplicidad y por su carácter dicotómico, fragmentado y desarticulado, acentuado por la escasez de habitantes. Tal situación los vuelve vulnerables ante los cambios. Las actividades dominantes son usos mono-funcionales que en su mayoría se desarrollan a escala doméstica y tradicional, a nivel de subsistencia, 0 cuando existe algún margen para la comercialización, se realiza a esa misma escala. Algunos de estos poblados están desarrollando experiencias en turismo. En cuanto a la Villa 21-24, posee complejidad en función del número de habitantes y la diversidad de actividades informales, aunque todo ello en un ambiente degradado.

Sinapsis: La situación de aislamiento a escala regional y la propia de escala local, impiden satisfacer este requisito de conectividad e interacción, acentuada por la escasez de habitantes, de diversidad de actividades y de redes de comunicación, constituyendo un aislamiento no solo físico, sino también respecto a redes de conocimiento. En la Villa 21-24, son valorables las interacciones que posee a nivel comunitario, internamente, así como con sus lugares de origen.

Sinergia: En función de lo expresado precedentemente, se reitera la falta de la masa crítica necesaria en cuanto a complejidad, diversidad de funciones y actividades, cantidad y composición de habitantes, cantidad y calidad de las conectividades e interacciones físicas, sociales y con las redes de información, para poder generar una reacción en cadena 0 efecto multiplicador en donde el todo sea más que la suma de las partes. La energía exógena necesaria -conocimiento, acción política- es insuficiente y con visión sectorial. La energía endógena se ve debilitada debido a la migración de la franja de población más dinámica y activa

\section{Recomendaciones}

-Dado que la mayoría de las condiciones para el DLS son deficitarias, es necesario internalizarlas en la cultura local, en sus organizaciones, sus habitantes y en los responsables de la gestión territorial.

- Las políticas de fortalecimiento de las economías regionales son un paso necesario, sin embargo, insuficientes, por su escala, para impulsar mejores condiciones de vida en el corto plazo.

-Se pone el acento entonces sobre la articulación, sobre las redes y la necesidad de intervenir sobre ciertos puntos del 
territorio como estrategia para potenciar a los poblados y sus comunidades.

Para alcanzar el nivel de complejidad requerido, además de identificar fortalezas locales, ventajas y posibilidades para nuevas actividades, cada poblado, con una visión eco-sistémica, deberá constituirse en nodo de una red de comunidades sostenibles organizada según el mix de la cultura del desarrollo, cooperación/competencia, compensando simplicidad y tiempo necesario para introducir complejidad, sinapsis y sinergia, mediante una interacción dinámica colaborativa, de cada uno de ellos en una red local, regional y nacional -red de redesco-gestionada entre los actores locales y las diferentes escalas de gobierno, estructurada a partir de un sistema diversificado de funciones, un sistema de conectividades físicas que posibiliten la movilización de personas y productos, un sistema de comunicaciones que posibilite la conexión con fuentes de información y conocimiento, todo ello de acuerdo a los condicionantes ecológico-culturales regionales, tecnologías apropiadas y contemplando a la cuenca hídrica como referente para la definición de las unidades territoriales en las que se incluyen y operan los nodos.

En esta red, los habitantes de los asentamientos marginales del AMBA, capitalizando interacciones, conocimientos y habilidades relacionados a sus lugares de origen, cumplirán un rol en la cadena productiva, ya sea en la propia producción, en el transporte 0 abriendo canales de comercialización y difusión de productos y de servicios de los nodos de la red, en mercados del AMBA, todo ello, acompañado desde la gestión pública.

Para avanzar en su operatividad, se recomiendan los siguientes pasos:

-Creación a escala nacional de una ley similar a la 13.251

de la Provincia de Buenos Aires - Régimen de Promoción de Pequeñas Localidades Bonaerenses, para promover y dar marco legal al desarrollo de la red de comunidades sostenibles.

-Promoción de ámbitos de gestión concertada en las diferentes localidades, creando o renovando un clima cooperativo y colaborativo, con educación para la toma de conciencia de su potencial.

-Definición de un modelo de desarrollo local/regional y de un perfil productivo claros, en función de lo que cada localidad/ región puede ofrecer, armando cadenas de valor asociadas -Estrategia de seguimiento y monitoreo de cada experiencia en particular y en su conjunto.

Para definir el modelo de desarrollo local/regional se enuncian las siguientes recomendaciones:

Promoción de actividades económicas locales/regionales - Transición del monocultivo a una creciente biodiversidad -Promoción de agroindustrias locales -artesanales, pequeñas y medianas- que agreguen valor a la producción primaria. Definición de nuevos sub-productos, con marcas que identifiquen su origen

-Desarrollo de micro, pequeñas y medianas industrias complementarias a las anteriores

-Explotación de las características climáticas de cada región para generar energía renovable.

-Promoción de la actividad turística cultural y natural. El capital cognitivo que poseen los habitantes originarios, posibilita que se formen como guías de turismo especializados. Deberá gestionarse la inclusión en circuitos turísticos más amplios. El turismo aventura, es también una opción.

\section{I+D - Formación de recursos humanos}

-Creación de una red de instituciones de investigación y universidades, para crear conocimiento sobre las posibilidades productivas de cada región y el mejoramiento de procesos y de gestión, en conexión con agentes públicos y equipos técnicos locales, así como para la capacitación de recursos humanos en tales temáticas y en otras -por ejemplo, en los oficios de los habitantes de la villa-.

\section{Conectividades}

-Tanto de transporte como de conocimientos, actuantes como factores de desarrollo.

-Expresado en puntos anteriores, tendiente a la conformación de la red de interacciones, tanto físicas, mediante las infraestructuras, modos y medios de movilización más adecuados para cada región: Ferroviario, fluvial, automotor, como la de redes informacionales, del punto anterior. Deberán contemplarse circuitos de transporte público escolar con móviles adaptados a las características de cada región, para garantizar el acceso a la educación

Equidad social

-Políticas públicas para asegurar igualdad de oportunidades 
para acceder a los beneficios del desarrollo, y para la solución de problemas -por ej, explotación cooperativa de predios rurales-

Es así que cada región deberá visualizarse como ámbito de desarrollo poli-nodal, equilibrado e integrado territorialmente, asegurando dinámicas de complementariedad generadoras de sinergias de proyección regional y nacional en función de sus capacidades locales, brindando oportunidades de progreso al revitalizar y potenciar actividades económicas diversificadas e innovadoras, en un clima de equidad social y sustentabilidad ambiental.

\section{Bibliografía}

- Benítez, Margarita. (2012) LaArgentinaquedesaparece. En Clarín Digital http://www.clarin.com/rural/expoagro/ pueblos-gente-desaparecieron_0_659934067.html

- Boisier, Sergio. (2004). El desarrollo territorial a partir de la construcción de capital sinergético.

- GCABA. (2009). Atlas de Indicadores de Desarrollo Urbano de la CABA. SSP-MDU-CABA

- Pasciaroni, C; Olea, M y Schroeder, R. (2010) Pequeñas localidades, entre el éxodo rural y la urbanización. Ponencia. VIII Congreso Latinoamericano de Sociología Rural, Porto de Galinhas. En http://www.oppad.uns. edu.ar/docs/trabajos/congresos/exodorural.pdf

- Reese, E. (2007). Paradigmas de desarrollo local en ciudades intermedias. Conferencia. $1^{0}$ Seminario "Instrumentos de Gestión para el desarrollo local en ciudades intermedias". Resistencia.

\section{Trabajos Prácticos elaborados por alumnos de la UM-} FADAU

- Carbó, Entre Ríos. Paola Cuervo, Daiana Rivero e Iván Gauto

- Las Marianas, Bs As. Estefanía Aguirre, Agustina Domenech, Agustín Baldo

- Mutquin, Catamarca. Tamara Sucko
- Pueblo Liebig, Entre Ríos. Virginia Maydana, Andrea Rodrigo y Natalia Valoni

- San Miguel de los Colorados, Jujuy. José Largües Caballero, Marcia Velázquez, Verónica Brautigham, Florencia Tomalino, Cristian Álvarez, Leandro Pinheiro.

- Villars, Bs As. Soledad de los Ángeles Álvarez, Javier Routin, Esteban Mateo

- Yacuy, Salta. Juan Manuel Morano, Roberto Fernández, Jazmín Montiel, Lucas Vilches, Patricio Echegaray, Daniel Garboni, Rodrigo Álvarez, Nicolás Mazzalupo

- Eje Riachuelo: Comunas 4 y 8, CABA. a.- Franco Cicco, Hernán Riccio, Julián Scocozza, Cintia Cáceres. b.Barmio, Barretto, Díaz

- Villa de emergencia 21-24, Barracas, CABA. a.Sergio Acevedo, Marcelo Alonso, Stefanía Pagliaro, Federico Birckenstaedt b.- Nazarena Aparicio, Marcelo Bertolusso, Franco Dell'Archiprete, Belén Menconi. c.- Vanesa Ríos,Stefanía Romano, Estefanía Álvez, Florencia Frick, Cristian Véliz d.- Carolina Cacciatore, Sabrina Cossani, Anahí Russo, Paula Gobbi 